\title{
Specify Other Narcotics or Opiates Administered
}

National Cancer Institute

\section{Source}

National Cancer Institute. Specify Other Narcotics or Opiates Administered. NCI

Thesaurus. Code C159298.

A request to specify narcotics or opiates that were administered but not included on a list. 